\title{
Opportunities and Challenges to Implementation of LIFI
}

\section{A Review}

\author{
Mohammad Abdullah', \\ Dept. of Software Engineering, \\ UOG Sialkot Campus, \\ abdullahxheikh03@gmail.com
}

\author{
Sameed ul Hussan², \\ Dept. of Software Engineering, \\ UOG Sialkot Campus, \\ sameedulhussan@gmail.com
}

\author{
Salman Safdar ${ }^{3}$, \\ Dept. of Software Engineering, \\ UOG Sialkot Campus, \\ 17241598-44@uogsialkot.edu.pk
}

$* * * * * * * * * * * * * *$

\begin{abstract}
LIFI was first introduce by Professor Harald Haas on July 2011. Light fidelity (LI-FI) may be a technology which uses light as a medium to travel from one place to a different. It uses Light like Light Emitting Diode (LED) visible radiation Communication (VLC) for data Transferring and Internet connection. LIFI may be a technique which uses light as a medium because it uses light to travel it's 20 times faster than any WIFI within the world. during this paper we speak about things like what's LIFI and the way it's better then WIFI why we want LIFI and What changes did bring LIFI in our Future LIFI. How it'll make IOT devices far better than before The Technique to implement LIFI and also the problem we face of implementation of LIFI.
\end{abstract}

Keywords: LIFI, WIFI, VLC, LED, IOT

\section{INTRODUCTION}

LIFI could be a wireless based system which uses Light Emitting Diode (LED) and visual Light communication (VLC) for travel from one place to a different. In simple words LIFI uses light as a medium to travel from one place to a different. LIFI was first introduce by Professor Harald Haas [1].In the upcoming Future where automobile devices Bicycle Motor bike Cars and even Doors are using Technologies which use Internet to control like ever car and motor bike encompasses a digital map on that which help him to travelled any unknown places easily. In 2019 Up to 10 billion Mobile devices exchange data and these are just mobile devices. Think if there was included BIG Data IOT devices and Other things. consistent with a pursuit in 2020 the quantity of things exceed up to twenty billion so you'll see what proportion is LIFI is very important in up comings days. As we all know WIFI uses radio active waves for transmission and LIFI uses light as a transmission medium so it had been much faster than WIFI. In WIFI there's a limit of what quantity we will send data at a time but in LIFI it had been nearly unlimited for data transfer in LIFI. In WIFI we use modem and wires for using of internet so after we are off from modem it made our file transferring so problematic but in LIFI we use LED light bulbs and tubes for as a replacement of modem briefly, every light bulb tube or the other light may be a source of Internet provider.

\section{LI-FI}

Light fidelity (LI-FI) you'll be able to say that it absolutely was the identical as WIFI if you say in simple terms because both are functioning on wireless principle both are used for transferring data and use of internet. LIFI was first introduce in 2011 by a professor of Harald Haas. They first introduce an idea where they use light to travel data from one place to a different. within the research they show that the sunshine is wont to travel data and it's 20 times faster and more stable than a standard WIFI system. In our up coming existence where time is money and that we must work faster then before 1 seconds can cause us such a lot loose so there we want a connection a service which is more reliable and faster which is more fascinating and who is more stable than the other service we've got. Visible light communications (VLC) was introduced by Tanaka, et al. [2] and uses high brightness white light-emitting diodes (LEDs) for digital communication. A limitation of LEDs is that the phase of the optical signal isn't available for data encoding. Therefore, VLC is proscribed to intensity modulation (IM)/direct detection (DD). Historically, this has limited the attainable transmission speeds. However, high speed data transmission with LEDs at low computational complexity was enabled by modifying multilevel and multicarrier modulation techniques [3]. LIFI relies in LED light. LED light can travel at a speed which in undetectable to human optic to transmit data. In facts it's been demonstrated that information is transmitted at the maximum amount as 224 gigabits per second [4], the equivalent of 18 movies of $1.5 \mathrm{~GB}$ each being downloaded every single second in lab conditions. you'll say that the guts of Li-fi technology is high brightness LED's LED may be switched on and off very quickly. you'll say in binary when LED in on that was 1 and if LED was off it had been 0 . and therefore the on off speed of a LED blub is one mini second 1?s. The importance of using VLC is that frequency above $3 \mathrm{THz}$ isn't regulated by radio regulating laws. WIFI which uses radioactive waves also are harmful to human. On the opposite hand LED Give us light take lesser energy than normal light bulb and that they are more brighter. Wi-Fi effects which are found by multiple Wi-Fi studies and are previously confirmed by non-thermal exposures to other microwave frequency EMFs. The 1971/72 U.S. Office of Naval Medical Research study (Glaser, 1971) reported the subsequent changes associated with testis or sperm [5]. 


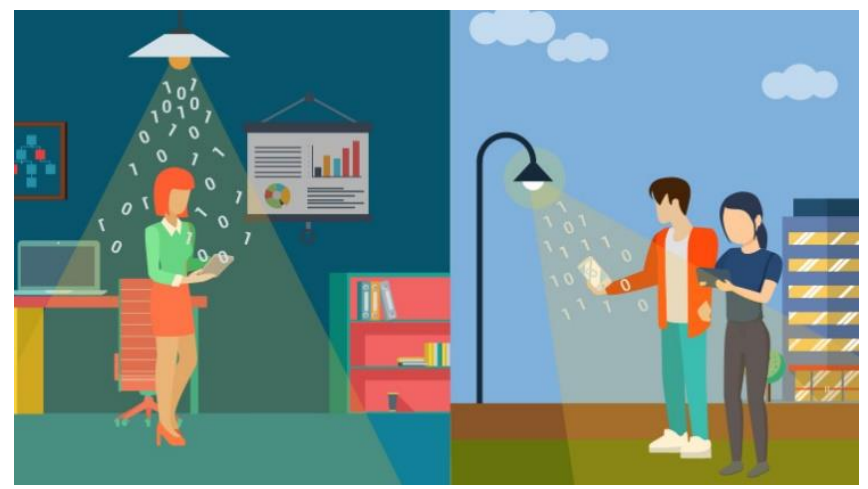

Fig 1.0 The Figure tell us about the LIFI system Work with the assistance of LED lights and blub. [6]

\section{INTERNET OF THINGS (IOT)}

As we find out about LIFI lets discuss a touch IOT internet of things. during this all the items are connected to at least one another with the assistance connection and that they are often control by the assistance of a sensor. The automated door Fig 2.0, Automatic lights are the instance of IOT. IN IOT we controlled of these things through internet or with the assistance of other wireless connection like Bluetooth. IOT devices make our life much easier and suitable for us and it also help us to not use extra energy for instance after we don't seem to be home all doors are automatically. So we don't need to go to every door to lock it.



Fig 2.0 A censoring door which detect the person in front of him and open Automatically with the help of sensors.

\section{LIFI IN INTERNET OF THINGS (IOT)}

AS on top of mention IOT devices uses sensing element and a web association and you'll build your whole house automatic like dextral Laboratory cartoon I sometimes watch after I was youngsters. thus once you build your whole house automatic if square measure|you're \} victimisation WiFi it'll additionally impact your health like on top of mention $\mathrm{WiFi}$ uses radio active waves and EFM wave that are thus harmful for human bodies internally and outwardly. thus LIFI is that the most suitable choice in IOT. LIFI is surroundings friendly. As mention wherever comes benefits there area unit disadvantages \{are additionally|also are|are\} there LIFI uses light-weight transmission thus it cannot passes through wall and in day there's an opportunity once you send signals it got distracted in SUN light-weight on the approach and it also troublesome for him to travel in traditional bulbs or lights.
LIFI primarily based machine-driven searching help application in IoT introduce by Sharmin Akter in 2018 [6]. wherever they use LIFI base system in IOT device that helps the client notice|to seek out|to search out\} there item simply and find the foremost things simply. it absolutely was created with 2 totally different version one internet and second mobile apps. Automatic charge system victimization LIFI was introduce by Zubin Thomas [7]. wherever he uses LIFI fir higher association in charge there product and System.

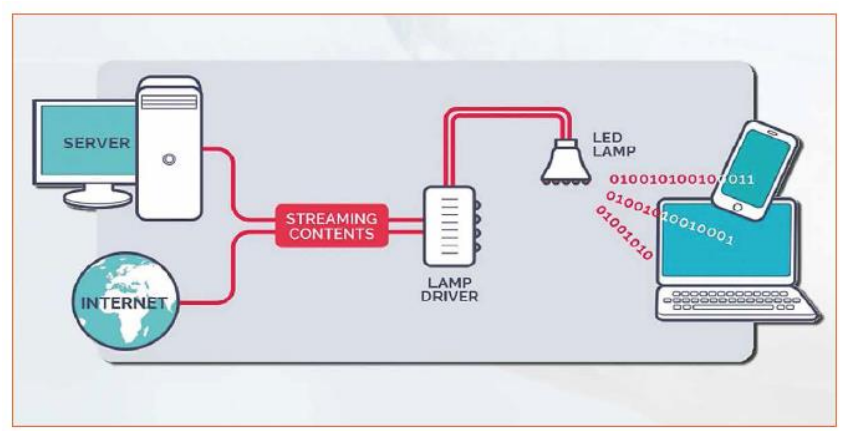

IV. IMPLEMENTATION OF LI-FI

There area unit several prospects wherever WiFi is best then LIFI however future lies in LIFI. wherever LIFI is incredibly helpful and it travel with quickest speed in whole world with the speed of sunshine it absolutely was additionally exhausting to implement everyplace and it's not a simple task. LIFI communication system involve full duplex communication mode. The downlink in LIFI use the actinic ray spectrum for transmission of by intensity modulation of LEDs in Solid State lightening system [8]. It uses A transmission wherever the most signals area unit coming back from so they travel through diode lights sort of a electronic equipment in WiFi. once LIFI was 1st introduce it absolutely was nearly not possible to implement LIFI instantly. so that they build it progress slowly.

\section{Fig 3.0 implementation of LIFI [6]}

LIFI technology is incredibly easy from a useful purpose of read. the bulk of applications exchange information in digital type. A transmitter, primarily Associate in Nursing diode, typically a optical maser, emits light-weight and knowledge at the same time. we have a tendency to then notice the quality components of \{a information|a knowledge|an information chain transmission: data, committal to writing and a network. These digital networks modulate the diode transmitter that then permits the transposition of the electrical signal into a light-weight signal. Then the sunshine signal is freely subtle into a space or outside. looking on the space, there's a bigger or lesser reduction and consistent with the assorted environmental disturbances (rain, sun, alternative lighting, etc.).A receiver, created from a photodetector or a camera upon arrival is chargeable for re-transcribing the lambent signal into Associate in Nursing operational electrical signal. including this conversion it's quite frequent particularly for top speed links to seek out the pre-ampLIFIcation level tailored to the specificities of the chain. The modulations used area unit primarily power modulations; the diode is thus piloted by a switch circuit supported easy physics. the chosen rates of modulation area unit sufficiently high thus on make sure that the aflicker isn't visible to the human eye ( $>$ two hundred $\mathrm{Hz}$ ) [9].

In the experiments performed by Herald Haas and his team, LEDs exhibited a possible to figure even once the receivers were part shaded. The experiments hint the viability of such systems in sensible readying and 
that they will be used for communication at nice speeds peaking to $1 \mathrm{Gbps}$ beneath clear conditions and quite

$100 \mathrm{Mpbs}$ in fog [8]. an entire LIFI network consists of relinquishment, multiple access, and co-channel interference (CCI) coordination, as shown in Fig. 4.0 There area unit 2 sorts of relinquishment: horizontal relinquishment and vertical handover. Horizontal relinquishment refers to a modification of the serving AP from among an equivalent radio access technology (RAT). Vertical relinquishment refers to a modification of the serving AP happiness to a special RAT. as an example, mobile users is also transferred from a LIFI AP to a Wi-Fi AP once none of the LIFI APs area unit able to supply a reliable link or the speed of the user is just too high so the dwell time in a very cell is just too short to ascertain a purposeful communication link. once the user slows down and enters the coverage of a gently loaded LIFI AP, it's going to be best to relinquishment to it LIFI AP to alleviate the Wi-Fi network for additional economical operation (e.g, guaranteeing less packet collisions). [10]

\section{Problem and solution in Different LIFI System}

In 2019 Ardimas Andi purwita introduce OFDM-base LIFI system in which they tells that how the FDM LIFI system work in indoor system [8]. MOHAMMAD DEHGHANI SOLTANI also work on OFDM error system in 2019 they analyze the device orientation and assess its importance on system performance. The reliability of an OWC channel highly depends on the availability and alignment of line-ofsight (LOS) links. In this study, the effect of receiver orientation including both polar and azimuth angles on the LOS channel gain are analyzed [11].in an another article S. Jayasudha also talk about the lifi effect on $5 \mathrm{~g}$ technology in that paper the tell us about a experiment A two minutes video is captured for each BER measure- ment to increase the BER reliability. In this proof of- concept experiment, only a single moderate brightness white-light LED is used, and the transmission distance at $\sim 500$ lux is $26 \mathrm{~cm}$. The transmission distance can be further enhanced by using higher brightness LEDs. The sensor cannot record any signal during the transfer time [see Fig. 1(b)], and this time in our camera is $14.29 \mathrm{~ms}$ ( $\sim 40 \%$ of an image frame). Hence, each data packet will be transmitted 3 times successively to ensure each image frame captured by the camera contains a complete data packet including both header and payload. Finally, the net data rate is $\sim 1 \mathrm{kbit} / \mathrm{s}$ with deducting the duplicated data packets [12].

\section{Conclusion}

Wireless communication Fast speed internet file transferring calling, video calling, and entertainment stuff now a days everything is related to mobile phone internet and wireless devices. As mention above there will be 20 billion mobile device in 2020 and its only mobile devices its not include laptop computer and other things. So we need fast speed and bigger network of internet. But wifi uses radio active waves and radio active waves are not only harmful for human health its also harmful for other animals trees and other living things so we need a network which is more less harmful for living things and that is LIFI LED and VLC base system which is Ecofriendly and 1000 times more faster then any wifi network available right now and it was the best solution and it was the future. We discussed some way to implement LIFI and see why it is difficult until now to implement it.
[1] H. Haas, "Properties if LIFI," What is LIFI, no. 2020, 2011.

[2] Y.Tanaka,T.Komine,S.Haruyama and M.Nakagawa, "Indoor Visible Communication Utilizing Plural White LEDs as Lighthining," 12th IEEE in Symp.on personal Indoor and Mobile Radio Communication, vol. 2, 2001.

[3] H. Haas, "Oppurtunity and Chanllenges of Future LIFI," no. 2019, pp. 1-2.

[4] A. Cuthbertson, "Li-Fi internet breakthrough:224Gbps connection broadcast with an LED bulb," no. Feb 2017.

[5] M. L.Pall, "Wi-Fi is an important threat to human health," Enviromental Research, vol. 164, no. 5, pp. 405-46, 2018.

[6] Sharmin Akter, Dr. Rashidah Funke Olanrewaju, Thouhedul Islam, Salma, "LiFi based automated shopping assistance application in IoT," Journal of Physics, no. 2018, pp. 1-6, 2017.

[7] Zubin Thomas, Nikil Kumar and D. Jyothi Preshiya, "Automatic Billing System using Li-Fi Module," no. 8, pp. 1-3, 2016.

[8] Ahsan Memon1*, Faisal Karim Shaikh2, Nafeesa Bohra2, Umair Jamil Ahmad3 and Mamta Bachani2, "Feasibility of LiFi in the Contemporary World," indian journal of Science and technology, no. 9, pp. 49, 2017.

[9] "LED Professional," LED professional Review, 2018.

[10] "Harald Haas, Liang Yin, Cheng Chen, Stefan Videv, Damian Parol,, "'Introduction to indoor networking concepts and," Journal of Optical Communications and Networking, vol. 12, no. February 2020, pp. 4-14, 2020.

[11] MOHAMMAD DEHGHANI SOLTANI, (Student Member, IEEE), ARDIMAS ANDI PURWITA,, "Impact of Device Orientation on Error," Journal of future issue, no. 9, pp. 1-4, 2019.

[12] S. Jayasudha, N. Bakkiyalakshmi, M. Manju, R. Sivabarani, M. Subasridevi, "Visible Light Communications For $5 \mathrm{~g}$ Wireless Networking Systems-Lifi," ADVANCES in NATURAL and APPLIED SCIENCES, vol. 4, no. 10 April, pp. 390395, 2016.

[13] Ardimas Andi Purwita, Mohammad Dehghani Soltani, Majid Safari, Harald Haas, "Impact of Terminal Orientation on Performance in," IEEE Wireless Communications and Networking Conference, no. 10, 2018.

[14] J. Ali, P. Youplao, K. Chaiwong, I. S. Amiri, S. Punthawanunt, N. Pornsuwancharoen \& P. Yupapin, "Broadband photon squeezing control using microring embedded gold grating for LiFi-quantum link," no. 12, 2019. 
[15] CHENG-LING YING, HAI-HAN LU, CHUNG-YI LI, CHUN-JEN CHENG, "20-Gbps optical LiFi transport system," OPTICS LETTER, vol. 40, no. 9, p. 14, 2015.

[16] Yunlu Wang, Dushyantha A. Basnayaka, Xiping Wu and Harald Haas, "Optimisation of Load Balancing in Hybrid LiFi/RF," IEEE Transactions on Communications, no. 5, 2018.

[17] H. Haas, "LiFi is a paradigm-shifting $5 \mathrm{G}$ technology," Reviews in Physics, vol. 3, pp. 26-31, 2018.

[18] A. R. Shrivas, "Li-Fi: The Future Bright Technology," International Journal of Electronics, Communication \& Soft Computing Science and Engineering,, 2015.

[19] N. Sarapat, Pornsuwancharoen, Youplao, M. A. Jalil, J. Al, "LiFi up-downlink conversion node model generated by inline," 2018 .

[20] E Ramadhani 1, G P Mahardika, "The Technology of LiFi: A Brief Introduction," Materials Science and Engineering, no. 18, 2017.

[21] Siyu Tao , Hongyi Yu, Qing Li and Yanqun Tang, "Performance analysis of gain ratio power allocation strategies for non-orthogonal multiple access in indoor visible light communication networks," EURASIP Journal onWireless Communications and, no. 10, 2018.

[22] HAI-HAN LU,* CHUNG-YI LI, CHIEN-AN CHU, TING-CHIEN LU, BO-RUI CHEN, CHANG-JEN WU, AND, "10 m/25 Gbps LiFi transmission system based on a two-stage injection-locked $680 \mathrm{~nm}$ VCSEL Transmitter," OPTICS LETTER, vol. 40, p. 19, 1 October 2015.

[23] Moussa Ayyash, Hany Elgala, Abdallah Khreishah, Volker Jungnickel, Thomas Little, Sihua Shao, Michael Rahaim,, "Coexistence of WiFi and LiFi Toward 5G Concepts, Opportunities, and Challenges," Optical Communications, February 2016.

[24] Dr. Naveen Rathee, Shreyaa Nagpal, Abhinav Malik, Charvi Khandelwal, "An Efficient Intelligent System for Data Communication Using LIFI Technology," INTERNATION JOURNAL OF COMPUTERS AND TECHNOLOGY, vol. 13, no. 10, p. 5044, 11 August 2014.

[25] Yunlu Wang and Harald Haas, "Dynamic Load Balancing with Handover in Hybrid," 2014.

[26] Gerardo Hernandez-Oregon,1,2 Mario E. RiveroAngeles ,1 Juan C. Chimal-Egu' 1a ,1, "Performance Analysis of V2V and V2I LiFi Communication," Wireless Communications and Mobile Computing, Vols. 1-12, 2019. 